\title{
Efecto de diferentes niveles de suministro de carbonato de calcio sobre el peso y grosor de la cascara del huevo
}

Effect of different levels of calcium carbonate supply on the weight and thickness of the egg shell

Vera-Rodríguez J, Hidalgo-Bravo G. Efecto de diferentes niveles de suministro de carbonato de calcio sobre el peso y grosor de la cascara del huevo. Rev Colombiana Cienc Anim. Recia. 2019; 11(2):Articulo719. DOI: https://doi.org/10.24188/recia.v11.n2.2019.719

Universidad de Sucre, Colombia

Los autores permiten a RECIA reimprimir el material publicado en él. En caso de que un autor quiera traducir o usar una publicación parcial o completa de nuestro Diario, el autor debe obtener un permiso por escrito del editor de la revista.

Revista Colombiana de Ciencia Animal - RECIA está distribuido bajo una Licencia Creative Commons Atribución-CompartirIgual 4.0 Internacional. 


\title{
Efecto de diferentes niveles de suministro de carbonato de calcio sobre el peso y grosor de la cascara del huevo
}

\author{
Effect of different levels of calcium carbonate supply on the weight and thickness of the \\ egg shell
}

José Humberto Vera Rodríguez M.Sc.

DOI: https://doi.org/10.24188/recia.v11.n2.2019.719

humbertorichi@hotmail.com

(D) https://orcid.org/0000-0003-3027-059X

Instituto Tecnológico Superior Enrique Noboa Arízaga.

Carrera en Tecnología Superior en Producción Agropecuaria.

Calle 4 de Noviembre y Loja. La Troncal, Cañar. Ecuador.

\author{
Gustavo Adolfo Hidalgo Bravo M.Sc. \\ turco1987@hotmail.com \\ https://orcid.org/0000-0001-7855-6402 \\ Instituto Tecnológico Superior Enrique Noboa Arízaga. \\ Carrera en Tecnología Superior en Producción Agropecuaria. \\ Calle 4 de Noviembre y Loja. La Troncal, Cañar. Ecuador.
}

Recepción: 30 Abril 2019
Aprobación: 18 Junio 2019
Publicación: 10 Julio 2019

\section{RESUMEN}

El objetivo fue evaluar el efecto de los niveles de suministro del carbonato de calcio $0.00 ; 0.50 ; 1.00$; $1.50 \mathrm{~g} / \mathrm{ave} /$ día, con una granulometría entre 2 a $4 \mathrm{~mm}$, al adicionarlos en las últimas horas de la tarde, sobre el peso del huevo y el grosor del cascarón en gallinas ponedoras de 22 a 30 semanas de edad. Se utilizaron 200 ponedoras de la línea genética Hy-Line Brown, las cuales fueron distribuidas en 4 tratamientos, 10 repeticiones, durante 9 semanas. Se evalúo semanalmente el peso del huevo y el espesor del cascarón, utilizando un diseño de bloques completamente al azar. Los resultados no registraron diferencias en cuanto al peso del huevo, mientras que para el grosor del cascarón se encontraron diferencias estadísticas significativas $\mathrm{p}<0.05$. En conclusión, se demostró que la adición

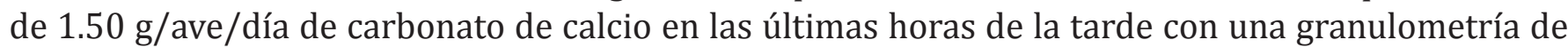
2 a 4 mm, mejoró el grosor del cascarón del huevo durante las semanas de evaluación, además se afirma que se puede estimar el grosor del cascarón del huevo a través del peso del huevo realizando el siguiente modelo de regresión (Grosor del cascarón = $0.3533 \times \ln$ (Peso del huevo) - 1.001).

Palabras clave: Avicultura, carbonato de calcio, grosor del cascarón, ponedoras, producción de huevos.

\begin{abstract}
The objective was to evaluate the effect of calcium carbonate supply levels $0.00 ; 0.50 ; 1.00 ; 1.50 \mathrm{~g} /$ bird/day, with a granulometry between 2 to $4 \mathrm{~mm}$, when added in the late afternoon, about the weight of the egg and the thickness of the shell in laying hens from 22 to 30 weeks of age. 200 layers of the Hy-Line Brown genetic line were used, which were distributed in 4 treatments, 10 repetitions, during 9 weeks. The egg weight and shell thickness were evaluated weekly, using a completely randomized
\end{abstract}


block design. The results did not show differences in the weight of the egg, while for the thickness of the shell significant statistical differences were found $p<0.05$. In conclusion, it was demonstrated that the addition of $1.50 \mathrm{~g} /$ bird / day of calcium carbonate in the late afternoon with a granulometry of 2 to $4 \mathrm{~mm}$, improved the thickness of the eggshell during the weeks of evaluation, It is also stated that the egg shell thickness can be estimated through the weight of the egg by performing the following regression model (Shell thickness $=0.3533 \mathrm{x} \ln ($ Egg weight) -1.001$)$.

Keywords: Poultry farming, calcium carbonate, shell thickness, layers, egg production.

\section{INTRODUCCIÓN}

La gallina Hy-Line Brown es la ponedora de huevo color marrón, considerada como una de las mayores productoras de huevos, ya que produce más de 467 huevos hasta las 100 semanas de edad, gallinas muy precoces al momento de iniciar la postura, tiene un buen pico de producción y un tamaño del huevo optimo (1). La selección genética ha modificado las características de las gallinas ponedoras, ahora son más productivas, con menor consumo de alimento, menor necesidad de mantenimiento, un mayor tamaño y peso del huevo (2).

Por tal motivo los que se dedican a la avicultura deben saber que para generar alimentos de buena calidad y bajo costos para el ser humano, y, en particular, el huevo de gallina que adquiere gran demanda por los consumidores; se debe suministrar fuentes de calcio a las gallinas, quienes puedan suplir sus exigencias diarias, especialmente al final de la recría y el inicio del período de producción, para que pueda optimizar sus reservas de calcio, en especial en aquellos momentos críticos en la vida de la ponedora (3).

El calcio es uno de los minerales esenciales en la alimentación de aves de corral, además de sus funciones vitales como el principal componente de la estructura ósea y la participación en el equilibrio ácido-base y el sistema enzimático, el calcio también es el elemento principal de la cáscara del huevo estimándose que cada huevo contiene alrededor de $2.2 \mathrm{~g}$ de calcio, presente principalmente en la cáscara del huevo; el calcio se lo suministra en los piensos ya que la mayoría de granos y sus insumos contienen niveles de calcio muy bajo (4).

La variación en la composición química, solubilidad in vitro, biodisponibilidad y granulometría son importantes para la selección de fuentes de calcio en la alimentación de las aves, la solubilidad es un factor principal, ya que está muy relacionada con la biodisponibilidad y absorción intestinal (5). Existen numerosos estudios destinados a determinar las necesidades de minerales, en especial de calcio, en las gallinas ponedoras debido que, desde el punto de vista económico, la avicultura se ve afectada por cuantiosas pérdidas debida a la fragilidad del cascarón, lo que incrementa el porcentaje de huevo roto (6).

Una de las posibilidades de poder manipular la calidad de la cáscara, es la utilización de fuentes de calcio grueso con una solubilidad entre 12-14\% y con un nivel de granulometría entre 2 a $4 \mathrm{~mm}$ para conseguir una liberación lenta del calcio (7). Los niveles de calcio en la dieta, granulometría y solubilidad de diversas fuentes de calcio son factores que influencian en la producción y calidad del cascarón del huevo $(8,9)$. Por este motivo, el objetivo de este trabajo fue medir el efecto de la adición de distintos niveles de carbonato de calcio durante las últimas horas de la tarde en la dieta de ponedoras de la línea genética Hy-Line Brown en fase I de producción, con un nivel de granulometría de 2 a 4 mm, sobre el peso del huevo y grosor del cascarón. 


\section{MATERIALES Y MÉTODOS}

Sitio de estudio. La investigación se llevó a cabo en la Región Tropical del Ecuador en la Avícola "SIRIA", ubicada en el sitio Mocochal de la parroquia Calceta cabecera cantonal del cantón Bolívar-

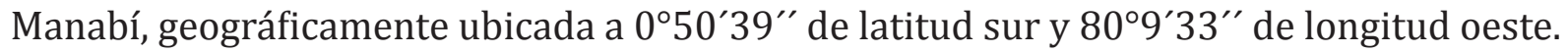

Manejo del experimento. Se utilizaron 200 ponedoras de la línea genética Hy-Line Brown en inicio de postura 22 a 30 semanas de edad, las cuales se encontraron separadas a razón de 5 aves por jaula. Las jaulas presentaron las siguientes dimensiones, $55 \mathrm{~cm}$ de ancho por $45 \mathrm{~cm}$ de largo, la maya que soporta los huevos es de $15 \mathrm{~cm}$ de largo para cada jaula. Las aves dispusieron de una dieta que se formuló cubriendo las necesidades de nutrientes de acuerdo al manual de manejo de ponedoras HyLine Brown (1) (Tabla 1). El alimento se suministró diariamente a las 7:00 de la mañana y el agua se ofreció ad libitum.

Tabla 1. Contenido nutricional de la dieta empleada en a las aves

\begin{tabular}{cc}
\hline Nutrientes & Valor nutricional \\
\hline Proteína & $17.35 \%$ \\
Energía & 320 EM.Kcal/ave/día \\
Calcio & $4.20 \%$ \\
Fosforo & $0.50 \%$ \\
Fibra & $3.80 \%$ \\
Sal & $0.20 \%$ \\
Lisina & $0.92 \%$ \\
Metionina & $0.45 \%$ \\
\hline
\end{tabular}

Durante la investigación se implementaron cuatro tratamientos cada uno con diez repeticiones: 0.50; $1.00 ; 1.50 \mathrm{~g} /$ ave/día de carbonato de calcio más un tratamiento testigo 0.00 al que no se le adicionó. La adición diaria de carbonato de calcio de origen de piedra caliza, se realizó con un nivel de granulometría de 2 a $4 \mathrm{~mm}$, entre las últimas horas de la tarde.

El rango del nivel de la granulometría del carbonato de calcio fue establecido tomando una muestra de 100 gramos, midiendo todas las partículas presentes con un micrómetro digital con un nivel de precisión 0-1" 0.00005" Marca FOWLER N 54-815 calibrado a un rango de hasta 2.5 centímetros.

Para establecer el peso del huevo se utilizó una balanza digital CAMRY EK5055 y para establecer el grosor del cascarón se tomó al azar semanalmente 10 huevos por tratamiento, para posteriormente romperlos y tomar una porción de la cáscara de la línea ecuatorial y medir con un micrómetro digital Marca FOWLER N 54-815.

Los resultados de los tratamientos se compararon mediante el análisis de varianza simple, empleando el diseño de Bloques Completamente al Azar, para las comparaciones entre las medias se aplicó en los casos requeridos la Prueba de Tukey con p<0.05. Para los cálculos se utilizó el Paquete Estadístico IBM SPSS versión 20. Además, fue utilizado el análisis de regresión para poder predecir el grosor del cascarón a partir del peso del huevo, realizando la siguiente ecuación: Grosor del cascarón $=(0.3533$ $\mathrm{x} \ln$ Peso del huevo - 1.001). 


\section{RESULTADOS}

Peso del huevo. En la Tabla 2 expone los resultados de la prueba de medias obtenidos en el análisis de varianza. En la variable peso del huevo no se encontró diferencias estadísticas significativas al adicionar los diferentes niveles de carbonato de calcio $0.50 ; 1.00 ; 1.50 \mathrm{~g} / \mathrm{ave} /$ día al ser comparadas con el tratamiento testigo durante las semanas de evaluación $\mathrm{p}<0.05$.

Tabla 2. Valores promedios de las variables peso del huevo y grosor del cascarón

\begin{tabular}{ccc}
\hline & \multicolumn{2}{c}{ Variable } \\
\cline { 2 - 3 } Tratamientos & Peso del huevo $(\mathbf{g})$ & Grosor del cascarón $(\mathbf{m m})$ \\
\cline { 2 - 3 } & NS & $0.41^{\mathrm{b}}$ \\
\hline 0,05 g/ave/día de carbonato de calcio & 56.15 & $0.42^{\mathrm{b}}$ \\
$1,00 \mathrm{~g} /$ ave/día de carbonato de calcio & 56.38 & $0.45^{\mathrm{a}}$ \\
$1,50 \mathrm{~g} /$ ave/día de carbonato de calcio & 56.64 & $0.40^{\mathrm{c}}$ \\
Testigo & 55.20 & 0.003 \\
EE & 0.52 & \\
\hline
\end{tabular}

a, b y c: letras distintas en una misma columna indican diferencias estadísticas según Tukey 0.05 .

NS No significativo. ${ }^{* *}$ Altamente significativo $\mathrm{p}<0.01$. EE Error estándar de la media.

a, b and c: different letters in the same column indicate statistical differences according to Tukey 0.05 . NS Not significant. ** Highly significant $\mathrm{p}<0.01$. EE The standard error of the media.

El tratamiento con mayor cantidad de carbonato de calcio 1.50 g/ave/día presentó un peso promedio de $56.64 \mathrm{~g}$, mientras que el testigo fue de $55.20 \mathrm{~g}$.

Grosor del cascarón. Los resultados del grosor del cascarón se aprecian en la Tabla 2. Se detectó una alta significancia estadística $\mathrm{p}<0.05$. El tratamiento con $1.50 \mathrm{~g} / \mathrm{ave} /$ día de carbonato de calcio fue el que mostró mejor promedio $0.45 \mathrm{~mm}$, en comparación con los otros tratamientos.

La Figura 1, muestra el análisis de regresión para las variables peso del huevo y grosor del cascarón para el tratamiento con $1.50 \mathrm{~g} / \mathrm{ave}$ /día que fue altamente significativo. Esta relación expresa que este modelo tiene un coeficiente de determinación de 0.81 para un total de 71 observaciones; el coeficiente de correlación tabular fue de $\mathrm{R}=0.90$, de tal manera que este modelo está asegurando un nivel de alta significancia estadística $\mathrm{p} \leq 0.0001$.

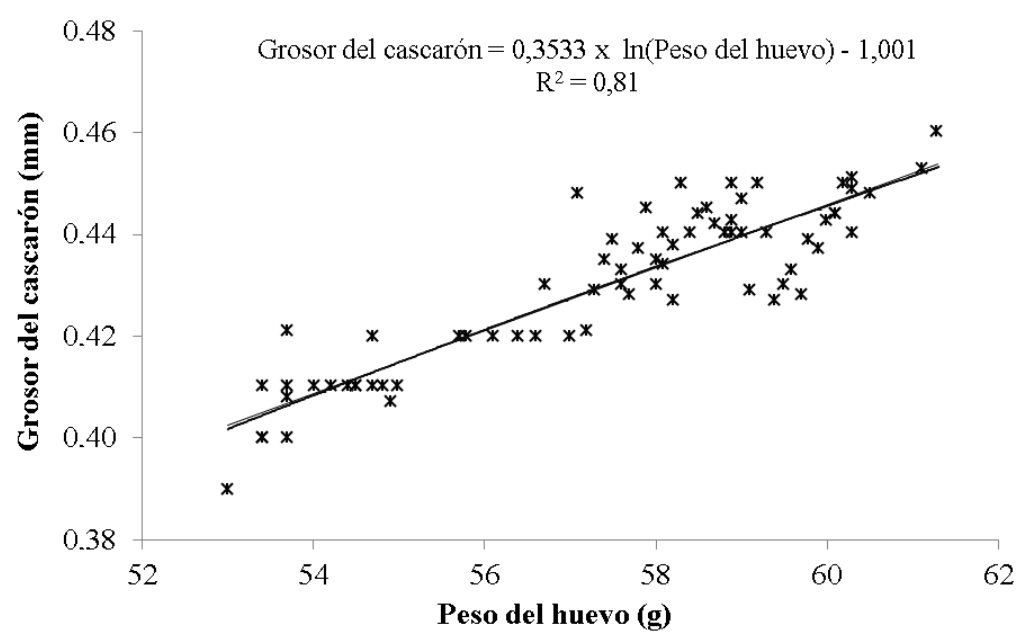

Figura 1. Análisis de regresión para las variables peso del huevo y grosor del cascarón en ponedoras Hy-Line Brown en inicio de puesta, bajo el efecto de $1.50 \mathrm{~g} / \mathrm{ave} /$ día de carbonato de calcio. 


\section{DISCUSIÓN}

Según Hy-Line Brown (1) y Hernández-Sánchez et al (16), el peso del huevo se ve limitado por el consumo de alimento y el contenido de aminoácidos presente en la dieta. Por tal, se debe reajustar las fases de alimentación en función de la proteína. Existe una fuerte correlación entre precocidad y peso medio del huevo, un peso corporal bajo en la madurez sexual reduce el peso medio del huevo $(10,11)$.

Por lo anterior, infieren Cuca (12) y Vera et al (13) que ponedoras de la línea genética Isa Brown alimentadas con carbonato de calcio con una adición de $1.50 \mathrm{~g} / \mathrm{ave} /$ día en horas de la tarde, no presentaron diferencias significativas al evaluar la variable peso del huevo con un promedio de 55.43 g. De igual forma Pizzolante et al (14) no encontró diferencias significativas en el grosor de la cáscara al evaluar dos niveles de calcio 3.5 y $4.0 \%$ con distintos tamaños de partículas en ponedoras Hy-Line Brown en II ciclo de producción.

Los resultados obtenidos por Safaa et al (15), indican que la influencia de distintos niveles de calcio en la dieta en el rendimiento productivo y calidad del huevo de ponedoras de 58 a 73 semanas de edad, demuestran que al aumentar el consumo de calcio se observa una mejora en el peso de la cáscara y grosor del cascarón. Estos reportes confirman la teoría de Hernández-Sánchez et al (16) quienes manifestaron que, el calcio es importante para una óptima producción y formación del cascarón del huevo porque un nivel inadecuado en la dieta de gallinas ponedoras puede afectar la calidad del cascarón y la producción de huevos. Además Lavelin et al (17) sostienen que los cascarones rotos o con fisuras causan pérdidas económicas a los productores de huevo.

Otro aspecto que es necesario medir, es la relación entre el peso de la cáscara y el peso total del huevo, pero sus medidas se deben tomar mediante un calibre de alta precisión y conviene hacer varias medidas para evitar valores sesgados (18). Es por tal motivo que no fue considera esta variable en la investigación.

En conclusión la adición de las diferentes dosis de carbonato de calcio en las últimas horas de la tarde en gallinas ponedoras Hy-Line Brown en inicio de postura, no influyó sobre el peso del huevo, pero si influenció sobre el aumento del grosor del cascarón, al adicionar $1.50 \mathrm{~g} / \mathrm{ave} /$ día de carbonato de calcio con una granulometría de 2 a $4 \mathrm{~mm}$. Conjuntamente, se puede estimar el grosor del cascarón a través del peso del huevo realizando el siguiente modelo de regresión (Grosor del cascarón $=0.3533$ $\mathrm{x} \ln ($ Peso del huevo) - 1.001).

\section{Conflicto de intereses}

Declaramos no tener conflictos de interés con respecto al trabajo presentado en este informe.

\section{REFERENCIAS}

1. Hy-Line Brown. La ciencia de la calidad del huevo. Boletín Técnico. 2017. URL: https://www. hyline.com/userdocs/pages/TU EQ SPN.pdf

2. Anderson KE, Tharrington JB, Curtis PA, Jones FT. Shell characteristics of eggs from historic strains of single comb white Leghorn chickens and the relationships of egg shape to shell strength. Int J Poult Sci. 2004; 3(1):17-19. http://dx.doi.org/10.3923/ijps.2004.17.19 
3. Campas DM. Sistema de alimentación por fase según variación en el nivel de proteína en dietas para ponedoras [Tesis]. Instituto de Investigaciones Avícolas, La Habana (Cuba). 2001.

4. Peixoto RR, Rutz F. Fontes de cálcio para poedeiras comerciais. I. Calcários "Matarazzo", "Trevo Filler" and "Trevo Dolomítico". Revista Brasileira de Zootecnia. 1988; 1:17-29.

5. Fassani EJ, Bertechini AG, Kato RK, Fialho ET, Geraldo A. Composição e solubilidade in vitro de calcarios calcíticos de minas gerais. Ciênc. Agrotec. 2004; 28(4):913-918. http://dx.doi. org/10.1590/S1413-70542004000400026

6. Sanmiguel-Plazas RA, Mejía-Rojas GR, Lozano-Covaleda LM, Castañeda-Serrano RD. Evaluación de diferentes granulometrías de calcio en la alimentación de gallinas ponedoras. Ciencia y Agricultura. 2016; 13(2):67-72. https://doi.org/10.19053/01228420.v13.n2.2016.5554

7. Zhang B, Coon CN. The relationship of calcium intake, source, size, solubility in vitro and in vivo, and gizzard limestone retention in laying hens. Poultry Sci. 1997; 76(12):1702-1706. https:// doi.org/10.1093/ps/76.12.1702

8. Rodríguez JHV, Pinargote MVV, Cedeño JOM. Adición de carbonato cálcico y su repercusión económica sobre el grosor del cascarón en ponedoras. Revista Espamciencia. 2012; 3(1):1-7. http://190.15.136.171/index.php/Revista ESPAMCIENCIA/article/view/46

9. Vera JH. Adición de carbonato cálcico en la dieta de ponedoras Isa Brown en inicio de la postura sobre el grosor de la cascara. Rev Cub Cienc Avíc. 2012. 36(1):69-70. https://doi.org/10.24275/ uami.3r074t937

10. ISA Brown. Guía de producción. Instituto de Selección Animal. 2017. URL: https://www.isapoultry.com/documents/37/IB cs c pguide L7150.pdf

11. López ÁG, Vivas A, Molina C, Rade D, Schnabel O. Diferencias observadas entre estándares internacionales de la gallina ponedora Isa Brown y los resultados obtenidos en el Cantón Bolívar, Ecuador. La Técnica: Revista de las Agrociencias. 2018; 19:75-90. https://doi.org/10.33936/ la tecnica.v0i19.822

12. Cuca GM. Estudios recientes con calcio en gallinas de postura. Programa de Ganadería, IREGEP. [En línea] Colegio de Postgraduados, Montecillo Estado de México. 2005: https://www. engormix.com/avicultura/articulos/estudios-recientes-con-calcio-t26033.htm

13. Vera RJH, Vélez PMV. Adición de carbonato de calcio en la dieta para mejorar los parámetros productivos en ponedoras Isa Brown Fase I. [Tesis]. Escuela Superior Politécnica de Manabí, Carrera Pecuaria: Bolivar, Ecuador, 2012. URL: http://repositorio.espam.edu.ec/ bitstream/42000/518/1/TMV57.pdf

14. Pizzolante CC, Saldanha ESPB, Laganá C, Kakimoto SK, Togashi CK. Effects of calcium levels and limestone particle size on the egg quality of semi-heavy layers in their second production cycle. Brazilian Journal of Poultry Sci. 2009; 11(2):79-86. https://doi.org/10.1590/s1516$\underline{635 \times 2009000200002}$ 
15. Safaa, H., Serrano, M., Valencia, D., Frikha, M., Moreno, E y Mateos, G. Productive performance and egg quality of Brown egg-laying hens in the late phase of production as influenced by level and source of calcium in the diet. Poultry Sci. 2008; 87(10):2043-2051. https://doi.org/10.3382/ ps.2008-00110

16. Hernández-Sánchez J, Cuca-García M, Pró-Martínez A, González-Alcorta M, Becerril-Pérez C. Nivel óptimo biológico y económico de calcio en gallinas Leghorn blancas de segundo ciclo de postura. Agrociencia. 2006; 40(1):49-57. https://www.colpos.mx/agrocien/Bimestral/2006/ ene-feb/art-5.pdf

17. Lavelin I, Meiri N, Pines M. New Insight in eggshell formation. Poult Sci. 2000; 79(7):1014-1017. https://doi.org/10.1093/ps/79.7.1014

18. Ortíz A, Mallo JJ. Factores que afectan a la calidad externa del huevo. Albéitar. 2013; 18-19. https://norel.net/es/system/files/factores que afectan_a la calidad del huevo 10.pdf 